\title{
Concept development of control system for perspective unmanned aerial vehicles
}

\author{
Vsevolod V. Koryanov ${ }^{1}$, Tatiana V. Kokuytseva ${ }^{1}$, Alexey G. Toporkov ${ }^{2}$, Stepan N. Iljukhin ${ }^{2}$, Il'ya O. Akimov ${ }^{2}$, Noa \\ Mohamado $^{2}$, Victoria Da-Poian ${ }^{2}$ \\ ${ }^{1}$ Peoples Friendship University of Russia (RUDN University), 117198, Miklukho-Maklaya str.6, Moscow, Russian Federation \\ ${ }^{2}$ Bauman Moscow State Technical University, 105005, 2-nd Baumanskaya, 5, b1, Moscow, Russian Federation
}

\begin{abstract}
Presented actual aspects of the development of the control system of unmanned aerial vehicles (UAVs) in the example of perspective. Because the current and future UAV oriented to implementation of a wide range of tasks, taking into account the use of several types of payload, in this paper discusses the general principles of construction of onboard control complex, in turn, a hardware implementation of the automatic control system has been implemented in the microcontroller Arduino platform and the Raspberry Pi. In addition, in the paper presents the most common and promising way to ensure the smooth and reliable communication of the command post with the UAV as well as to the ways of parry considered and abnormal situations.
\end{abstract}

\section{Introduction}

Currently the problem of the creation of unmanned aerial vehicles (UAV) with different function and configuration is extremely urgent. Different applications areas can benefit of this new technology. Civilian UAVs are used for different purpose such as aerial photography, patrolling areas, inspections, delivery of goods, supply of communication with designated areas, control of airspace and many other areas.

Thanks to modern conditions, the practical component in the creation of a UAV design and its control systems is not anymore a complicated science-intensive process such as previously.

Though, the UAV control systems have identical morphology, but due to the various purpose of the aircraft mission, the architecture of their control system (governing bodies, structure of onboard systems, communication system, sensor equipment and so on) can be created in different way to fulfil specific objectives.

Therefore, it is possible to mark the following urgent tasks, which will be solved in the development process of management systems:

- simplicity and reliability of construction;

- simplicity of operation in service;

- low competitive cost;

- insignificant mass-dimensional characteristics;

- enough high universality which will provide a possibility of placement on other types of aircraft including piloted;

- high level of fail safety of system to emergency situations.

\section{Appearance perspective UAVs}

In Moscow State Technical University of N. E. Bauman, within research and development activities operation over various youth projects, one project is about the carried "Sub-orbital rocket plane" [1]. As an example, this project is especially interesting thanks to the management systems: it was created "from scratch", without use of expert estimation elements, in a type of absence of a sufficient array of the statistical data obtained in an analysis result of properties of the existing samples. It is also worth noting that, unlike the majority of amateur projects about the creation of UAV, this one focuses not only on the structuration and synthesis of the command, but also combined management systems were made.

The sub-orbital rocket plane represents the perspective reusable UAV that serves for deduction of the disposable loads on height of low orbits of artificial Earth satellites, carrying out research experiences in the conditions of microgravity, execution of functions of communication and remote sensing of Earth. One of the UAV configuration is shown in Figure 1.

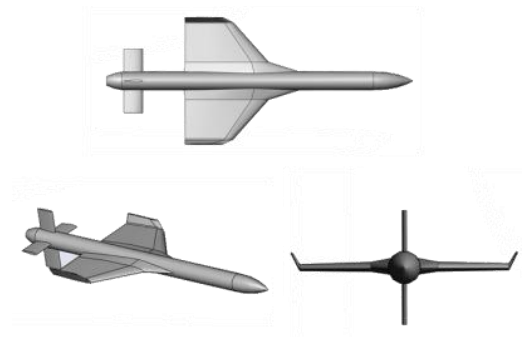

Figure 1. Various forms of UAV 
The process of correcting the UAVs aerodynamic model from aerodynamic tests data was studied. The task of identifying the UAV aerodynamic model can be summarized as the correlation between the initial aerodynamic model and the data of aerodynamic tests [2]. This task consists in the optimization of the design solution for regularity criteria. The number of these criteria is equal to the number of calculated aerodynamic coefficients.

The task of assessing the completeness of the UAV aerodynamic model is to select its structure of an aerodynamic model with the simultaneous selection for each structure of optimal aerodynamic derivatives.

Experimental studies were preceded by analytical calculation and computer simulation. In this paper, the results of mathematical modeling for UAV subsonic flow are presented. Calculations were performed using specialized software: SolidWorks (Flow Simulation) and ANSYS CFX. Then, the results were compared with the results obtained in the analytical calculation.

An example of flow visualization used to determine the value of on UAS drag's coefficient is shown in Figure 2 .

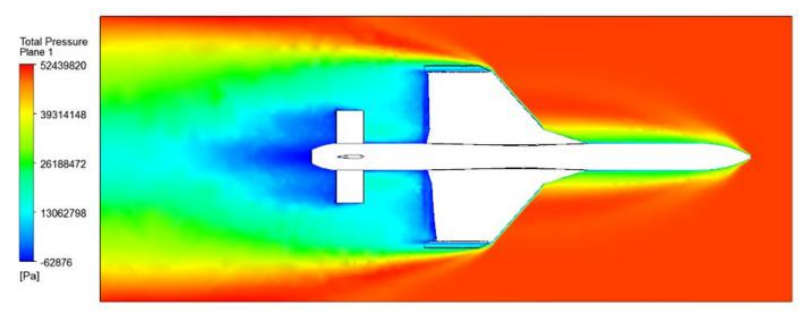

Figure 2. Example determining drag's coefficient

An analytical calculation was carried out according to the principle of the "UAV dismemberment" into isolated elements. Using this principle, the UAV was replaced by a designed scheme, in which the following elements were singled out: an isolated wing, an isolated aft body and an isolated hull.

The rocket plane, like any automatic aircraft, has a row of subsystems of the lowest hierarchical level as a part of the management system (see Figure 3). These includes: sensitive elements (MEMS sensors, temperature sensors, strain gages), electric power supplies of onboard systems, control moment gyroscope (two-degree force gyrostabilizers), the executive mechanisms (mechanism of disclosure of a wing, pyrosystem, servo actuators), and a basic element projected management systems - the onboard complex of control.

\section{Emergency situations}

As the UAVs have a long autonomy of flight and uses for a long time an autonomous mode without pilot tasks it can potentially constitute a threat for people near settlements, for infrastructure or for industrial facilities. Besides payload, the UAV capacity can be a special value, as well as the construction of the aircraft. For these reasons, the uncontrollable fall probability of the device is minimized, and the efficiency of the execution of the work is closer possible by all means and methods. Emergency situations have been studied in detail by NASA after communication issues [3], and unmanned missions emergency situations are still one main concern.

For the modern aircraft, the greatest danger is constituted by complex influence of operational factors, such as:

- failures of mechanical nodes of the aircraft;

- errors of operation of onboard systems at the level of algorithms and logic of functioning;

- the strong wind loads, in particular turbulence of the environment;

- rain, freezing and other meteorological factors;

- other factors.

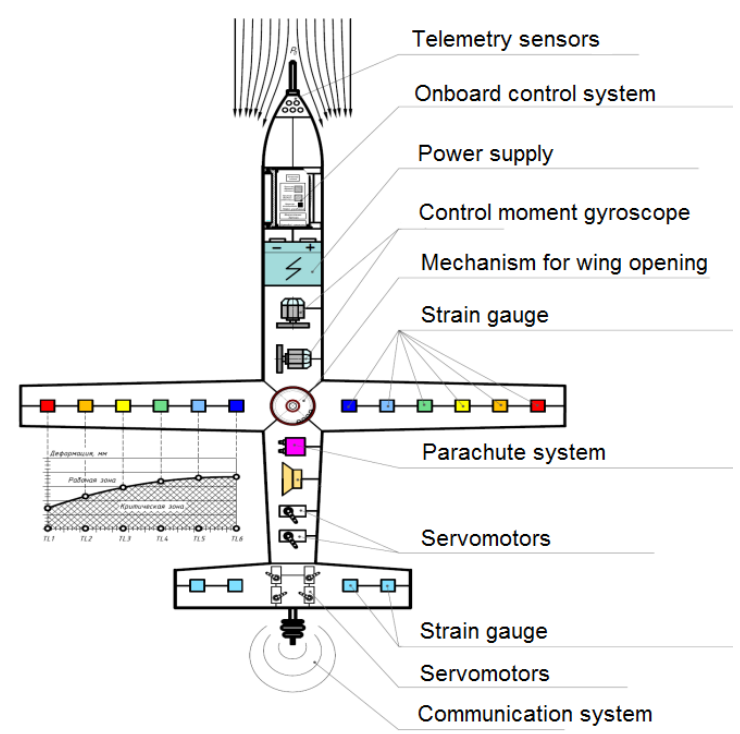

Figure 3. Composition of the UAVs onboard systems

Therefore, it is appropriate to consider an original problem of an emergency situation for two essentially different cases.

In cases of loss of communication with ground stations, the UAV belong to the first case. The normal mode is activated by implementing off-line control. In that case, special laws of control are used and the aircraft executes some implemented programs of control, and also realizes some algorithms in order to return the aircraft to a cover zone where signals of ground station control are provided.

In the second case, also with a loss of communication, certain emergency situations can emerge. It is known that compared to flight plan, these situations can be separated on considered and unconsidered emergency situations [4]. The considered emergency situations are those which probability of appearance was defined during flight planning stage. In this case, it is possible to write programs and algorithms of onboard systems operations in order to respond to these mergency situations.

The development of the instructions to avoid these considered emergency situations begins with compilation of the entire database of all aircraft subsystems, up to specific nodes and details. Then statistic analysis of probabilities and the reasons of their possible failures are 
carried out. Besides, development of techniques of detection of possible breakage and a technique of problematic situations removal are necessary.

During the flight of the perspective UAV, emergency situations will be removed in 3 steps. If operations at the current step do not allow to fix the problem, the process proceeds to the next step.:

1) Algorithms are guided by the indications of the sensors implemented in different systems. These sensors reveal the emergency modes and try to find instructions to eliminate the emergency situation found.

2) The algorithm removes the most likely causes of a problem. At the same time, the basis of the considered emergency situations ranked by probability of origin is used.

3) The algorithm adjusts to an emergency situation reducing as much as possible negative effect of its existence.

If the provided algorithms do not lead to the elimination of an emergency situation, it is then necessary to adapt the management system under the current conditions. In particular, to ensure the aircraft flight without using the faulty component, it is necessary to unify as much as possible the key algorithm control of the aircraft - a stabilization algorithm.

\section{Management system movement and navigation}

UAVs technology requires to analyse the relationship between the flight behaviour and all the technical parameters of the components the vehicle is made of [5]. Any effective system of automatic control represents a follow-up circuit. UAVs will be designed using optimal architecture system for future missions in order to benefit from advanced technologies and development facilities [6]. As a follow-up circuit, each such system possesses a certain set of measuring means. The system of angular stabilizing is based on the PID-regulator (Proportional Integral Derivative) which operation quality directly depends on the accuracy of its given coefficients. The perspective direction of development of autonomous systems of control are the adaptation of systems of control. For instance, under different weather conditions these coefficients will have different values. Also, if the UAV transports loads have different weight and overall dimensions, then these PID-regulator coefficients will also differ. In that sense, automated setup of "PIDcoefficients" was applied. Such setup has to be made directly during the flight by the analysis of the UAV response to the available perturbations. This setup must be performed either at certain time intervals, either in case of essential change of the external environment parameters.

In order to deal with the task of navigation, information from the sensors GPS and GLONASS which is filtered and analyzed in the flight program are used. These navigation data are processed by an algorithm, and, according to the flight job, teams on governing bodies for aircraft orientation are formed. In a suborbital rocket plane MEMS - the following sensors represented in the
Figure 4 are used: accelerometers, gyroscopes and compasses. The data obtained with these sensors are exposed to the linear filtering using Kallman's filter. To eliminate the accumulated error due to integration of data of a gyroscope, the complementary filter adding data of a gyroscope indexes of the accelerometer and calculating the true orientation angles of the aircraft is used. Also, the sensors located in different onboard systems and nodes, which allow to trace an aircraft time status at the moment are used. For instance, the strain gages set in a wing allow to trace its intense deformed status, and the differential pressure sensor (the pressure sensor of a filling flow allowing to determine aircraft speed) allows to adjust indications of the inertial MEMS - sensors and the GPS sensors monitor the aircraft orientation and its center of masses position.
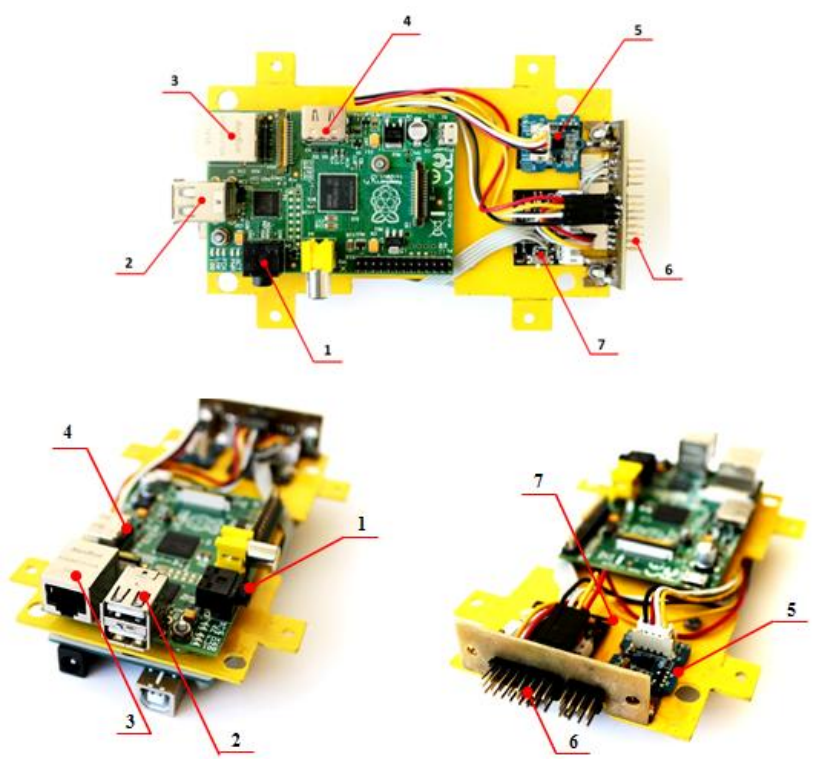

Figure 4. Experimental module of management system movement

1 - connector of the search and rescue device; 2 USB; 3 - Ethernet; 4 - "Output" for debugging of the program of the onboard electro-computer; 5 - gyroscope; 6 - connector of control of the periphery; 7 accelerometer.

In the case of hardware implementation of automatic control systems in this project, widely used microcontroller platforms, such as Arduino and Raspberry Pi are used. These platforms provide a fairly simple and intuitive interoperability layer with the programmer. Moreover, they have small weight, developed infrastructure and good productivity. In particular, Arduino [7] is used for the unit of stabilization system where the platform integrates sensors datas and, according to control box commands, gives the controlling impacts on executive bodies for orientation on the scheduled course. Functions of remaining units are performed, instantly, by Raspberry Pi as this platform has a processor with high clock rate and uses the Linux operating system.

The main characteristics of the developed module of management system movement are shown in the Figure 5. 


\section{Main characteristics}

\begin{tabular}{|l|l|}
\hline Supply voltage: & $6 . .15 \mathrm{~V}$ \\
\hline Power consumption (with sensors): & $<150 \mathrm{ma}$ \\
\hline Dimensions: & $147 \times 77 \times 47 \mathrm{~mm}$ \\
\hline Weight (with servo drives): & $180 \mathrm{~g}$. \\
\hline
\end{tabular}

Inputs and outputs:

- 6 PPM channels for connection to the RC receiver

- 6 PPM output channels for connection to servo drives

- USB connector for PC connection

- SPI Connector

- I2C connector

- UART (GPS signal)

- Analog inputs

Figure 5. Main characteristics module of management system movement

Navigation of UAV. To navigate the information from the GPS / GLONASS sensors are used. Their data are further filtered and analyzed in the flight program. Information from the GPS-sensor is compared with the information obtained by integrating the readings of inertial sensors (Gyrometer sensor and accelerometer). The architecture of the navigation system is shown in Figure 6.

Also, when creating the navigation algorithm, the initial data of the NoFlyZone.org project was used and now, data from this portal can be downloaded to the base of the onboard control complex. Also in the flight program, the areas forbidden for flight can be set.

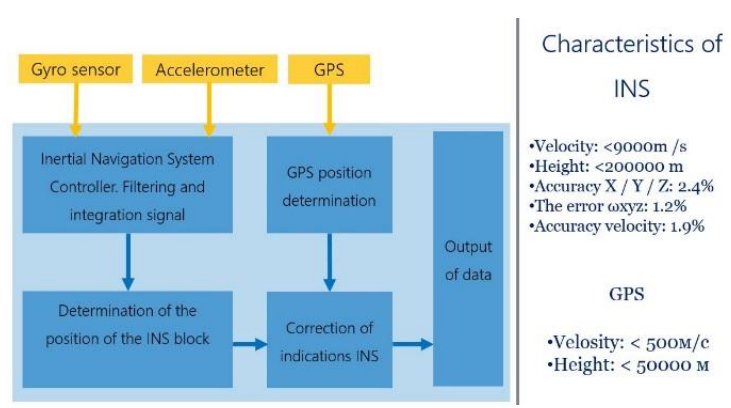

Figure 6. Navigation system

Stabilization of UAV. For the stabilization, inertial sensors are used: Gyro sensor and accelerometers. To correct the sensor readings, an incident pressure sensor is used to calculate the speed of the aircraft. The architecture of the stabilization system is shown in Figure 7.

Information from the sensors is processed by algorithms of control, which then send commands to the controls for the aircraft orientation at a given mission.

As mentioned above, we use MEMS sensors: accelerometers, gyroscopes and compasses (Figure 4). Data coming from MEMS sensors are processed by the Kalman filter (Figure 8). To eliminate the accumulated error of integrating the gyro data, a complementary filter that complements the gyro data with the accelerometer data is used and calculates the true angles of the aircraft orientation.

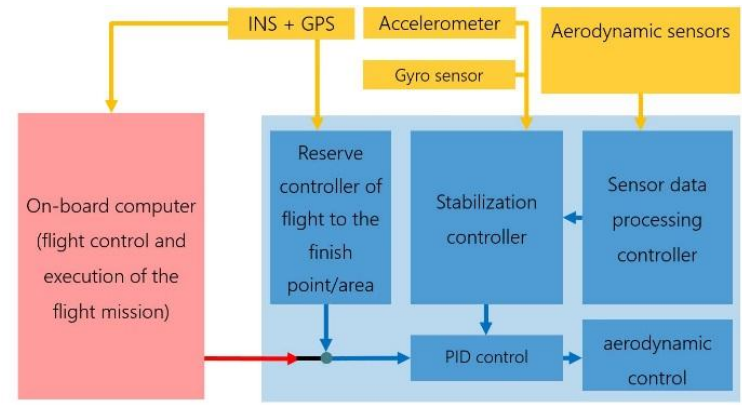

Figure 7. Stabilization system

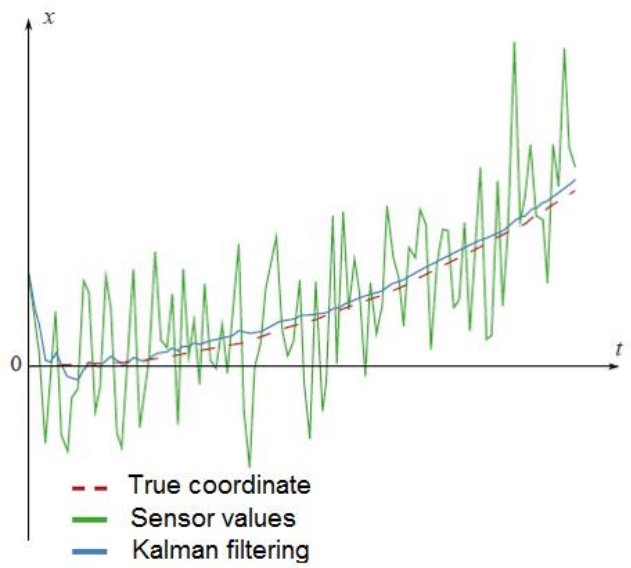

Figure 8. Kalman filter

Automated simulation stand. In order to check the software and the hardware of the UAVs automatic control system (ACS) and in order to timely detect errors, an automated simulation element was developed. This element is implemented on the basis of scripts operating in the MATLAB system running an IBM PC-compatible computer. This element is interfaced with the hardware module of the ACS via the USB interface.

Simulation element implements the process of controlling the model movement of the control object (UAV) under the control of the connected ACS. It is possible to specify various initial conditions and modeling scenarios, including simulating the occurrence of abnormal situations. At this stage, simulation provides: testing the operability of the hardware ACS modules; simulation of UAV motion with imitation of various abnormal situations and disturbing effects; exchange of information with the USB interface; reception, recording, processing and visualization of control signals generated by the ACS.

Simulation modeling and testing of the main systems and components of the experimental module was carried out using Matlab (see Figure 9). On the right of the figure, graphs of changes in the yaw, roll and pitch angles are illustrated.

These platforms have an additional advantage: during their existence, the big array of practical data and algorithms available to adaptation of the comparative analysis appeared. 


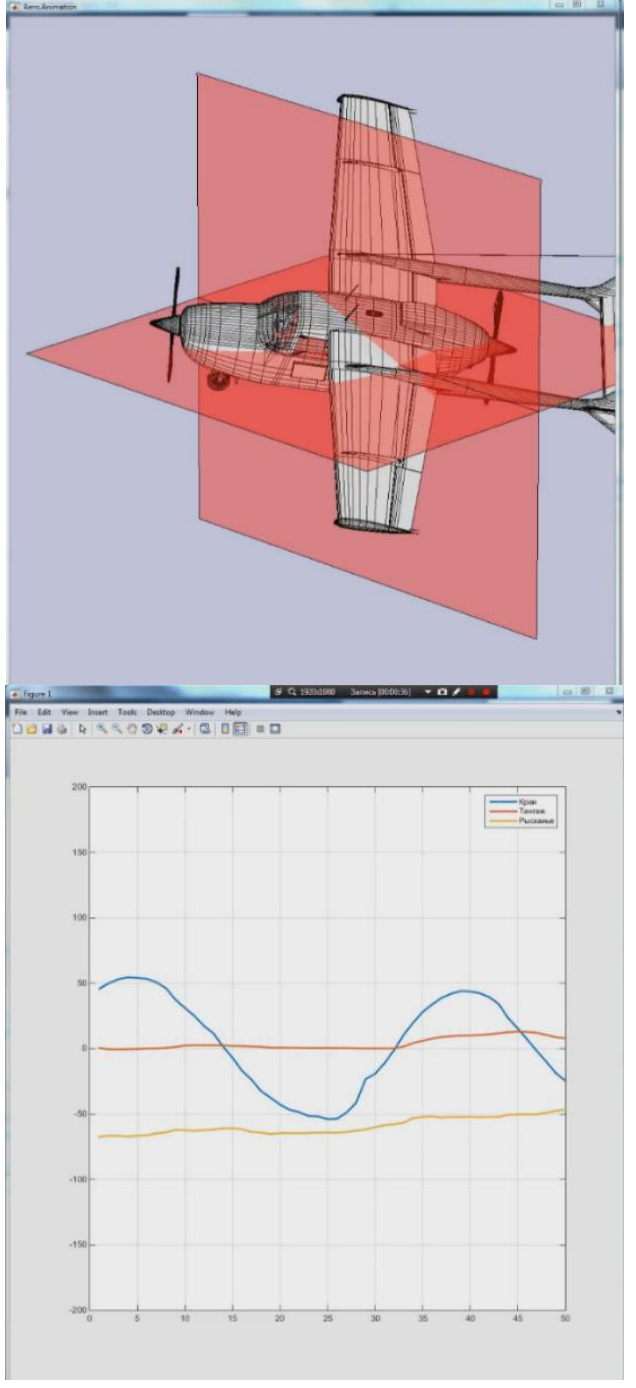

Figure 9. Simulation using Matlab

\section{Conclusions}

In operation, the main aspects of the UAV management systems for the example of a specific project are provided. At this stage of development, the management system has the following properties:

1. Quasiautonomy. Allows to minimize the involvement of the ground control station operator from take-off until the successful landing taking into account the origin of an emergency situation.

2. Modularity. The modular architecture allows to change and expand if necessary quickly a configuration and a functionality of system, thereby timely to increase fail safety of all control object in general, and also to reduce mass-dimensional and cost characteristics. The most important in case of operation of management system on different devices in different weather conditions taking into account requirements of the customer.
3. Universality. The system was developed for a suborbital rocket plane which could have different wing constructive implementations, however at this stage the developed system provides quasi-automatic control of the UAV weighing up to a 10 kilograms aircraft only with the fixed position of a wing for which flight tests were carried out. In the longer term regarding aircraft, the system will be able to control devices of helicopter type, multirotor systems, autogyros... The architecture and modularity of system will allow to test architectures, and system's modularity will allow to exercise further object managements not only terrestrial, but also maritime

\section{References}

1. Toporkov A.G., Mullin N.A., Pavlov N.G. Innovatsionnyy proyekt "Suborbital'nyy raketoplan» [Innovation Project "Suborbital rocket plane"]. Aktual'nyye problemy kosmonavtiki: Trudy XXXIX akademicheskikh chteniy po kosmonavtike, posvyashchennykh pamyati akademika S.P. Koroleva i drugikh vydayushchikhsya otechestvennykh uchenykh [Actual problems of space exploration: proceedings XXXIX Academic Space Conference dedicated to the memory of academician Korolev S.P. and other prominent russian scientists]. Moscow, BMSTU Publ., 2015, pp. 367-368 (in Russ).

2. Koryanov V.V., Nguen K.T., Nguen V.T. Multiple Regression for UAV Aerodynamic Model Based on Experimental Data. Vestn. Mosk. Gos. Tekh. Univ. im. N.E. Baumana, Mashinostr. [Herald of the Bauman Moscow State Tech. Univ., Mech. Eng.], 2016, no. 5, pp. 59-67. DOI: 10.18698/0236-39412016-5-59-67 (in Russ).

3. Manned spacecraft center NASA. Results of the second US manned suborbital space flight, 1961.

4. Solovyev V.A., Lysenko L.N., Lyubinskiy V.Ye. Upravleniye kosmicheskimi poletami. Chast' 2 [Control of space flights. Part 2]. Moscow, BMSTU Publ., 2010, part 2, 426 p. (in Russ).

5. Garcia Gallego, Gonzalo Augustin. Development of a model-assisted approach for the requirement-driven design and optimization of a quadrotor unmanned aerial vehicle, 2016.

6. Daniel Selva. A concept for an Agile Mission Development Facililty for CubeSat and suborbital missions, IEEE, 2016.

7. Jeremy Blum. Exploring Arduino: Tools and Techniques for Engineering Wizardry, Wiley, 2013, $384 \mathrm{p}$.

The publication was prepared with support of the «RUDN University Program 5-100». 